\title{
Resilient ZnO nanowires in an irradiation environment: an in situ study
}

\author{
C. Sun ${ }^{1,2}$, B. P. Uberuaga ${ }^{2}$, L. Yin ${ }^{3}$, J. Li ${ }^{1}$, Y. Chen ${ }^{1}$, M. A. Kirk ${ }^{4}$, M. $\mathrm{Li}^{5}$, \\ S. A. Maloy ${ }^{2}$, H. Wang ${ }^{6}$, C. Yu ${ }^{3}$ and X. Zhang ${ }^{1,3 *}$ \\ ${ }^{1}$ Department of Materials Science and Engineering, Texas A\&M University, College Station, TX \\ 77843 \\ ${ }^{2}$ Materials Science and Technology Division, Los Alamos National Laboratory, Los Alamos, \\ NM, 87545 \\ ${ }^{3}$ Department of Mechanical Engineering, Texas A\&M University, College Station, TX 77843 \\ ${ }^{4}$ Materials Science Division, Argonne National Laboratory, Argonne, IL 60439 \\ ${ }^{5}$ Nuclear Engineering Division, Argonne National Laboratory, Argonne, IL 60439 \\ ${ }^{6}$ Department of Electrical and Computer Engineering, Texas A\&M University, College Station, \\ TX 77843-3123
}

*Corresponding author: X. Zhang, zhangx @tamu.edu, Tel: (979) 845-2143 


\begin{abstract}
:
$\mathrm{ZnO}$ nanowires (NWs) have been extensively studied for various device applications. Although these nanowires are often suspected to be impractical and highly unstable under hostile radiation environments, to date little is known on their radiation tolerance. Here, we show outstanding resilience of $\mathrm{ZnO} \mathrm{NWs}$ by using in situ $\mathrm{Kr}$ ion irradiation at room temperature inside a transmission electron microscope. Our studies show that $\mathrm{ZnO}$ nanowires with certain diameter become nearly immune to radiation damage due to the existence of dislocation loop denuded zones. A remarkable size effect also holds: the smaller the nanowire diameter, the lower the defect density. Rate theory modeling suggests that the size effect arises from fast interstitial migration and a limit in size to which interstitial loops can grow. In situ studies also revealed a surprising phenomenon: the pristine prismatic loops can prevail over the strongest known defect sinks, free surfaces, to trap radiation-induced defect clusters. This study comprises the first critical step towards in-depth understanding of radiation response of functional oxide nanowires for electronic device applications in extreme environments.
\end{abstract}

Keywords: ZnO nanowires, radiation damage, extreme environments, defect denuded zone 


\section{Introduction}

Nuclear energy provides more than $10 \%$ of electricity internationally and is increasingly used in developing countries. Development of next generation nuclear reactors requires radiation tolerant ceramics for various applications, including electronic devices, sensors, immobilization forms for radioactive wastes, cladding materials for gas-cooled fission reactors and structural components for fusion reactors [1-3]. Under heavy ion irradiation, the primary damage in ceramics is the displacement of atoms/ions and the consequent formation of Frenkel (interstitialvacancy) pairs and defect clusters. The formation and migration of defect clusters in irradiated ceramics are complicated due to the presence of multiple sublattices, prominent bond orientation effects, electrostatic effects and greater importance of chemical and ionization effects [4].

Dislocation loops and voids are two major types of defect clusters formed in irradiated ceramics. In $\mathrm{Zr}^{+}$ion irradiated $\mathrm{MgAl}_{2} \mathrm{O}_{4}$ at $650^{\circ} \mathrm{C}$, interstitial loops formed on $\{11\}$ and $\{110\}$ planes, with Burgers vector along $<110>$ direction [5]. Certain oxides, such as magnesia $(\mathrm{MgO})$ and alumina $\left(\mathrm{Al}_{2} \mathrm{O}_{3}\right)$, are susceptible to formation of voids $[4,6]$. Defect denuded zones (DDZs) in the vicinity of defect sinks are typically observed after radiation at high temperature. For instance, DDZs along grain boundaries (GBs) were reported in SiC after neutron irradiation at $1010-1380^{\circ} \mathrm{C}$ [7]. Under light or heavy ion irradiation at $650^{\circ} \mathrm{C}$, DDZs formed near the surface or adjacent to GBs in $\mathrm{MgAl}_{2} \mathrm{O}_{4}$ and $\mathrm{Al}_{2} \mathrm{O}_{3}$ [5]. Volumetric swelling due to accumulation of point defects, defect clusters (dislocation loops, voids, etc.), or amorphization [2, 8, 9] has been frequently reported in various irradiated ceramic materials [10-13]. Unit-cell volume expansion was reported in $\mathrm{Ar}^{+}$ion irradiated polycrystalline $\mathrm{Ca}_{2} \mathrm{Nd}\left(\mathrm{SiO}_{4}\right)_{6} \mathrm{O}_{2}$ [8]. Macroscopic volumetric swelling reached $18.4 \%$ in zircon $(\mathrm{ZrSiO} 4)$ under alpha decay and was attributed to radiationinduced amorphization [14]. 
Strategies that introduce defect sinks, such as GBs [15-17], phase boundaries [18, 19], twin boundaries [20] and immiscible layer interfaces[21], have been applied to suppress the formation of defect clusters in metals and ceramics. Free surfaces are the strongest known defect sinks and nanoporous metals have shown superior radiation tolerance [22, 23]. A recent research showed helium-implanted $\mathrm{Ni}_{73} \mathrm{P}_{27}$ metallic glass nanocylinders exhibit enhanced ductility without sacrifice in yield and ultimate tensile strength [24]. ZnO NWs have been extensively investigated for electronic device applications [25-27] and could be a representative device candidate for in nuclear reactors.

Here, by using in situ $\mathrm{Kr}$ ion irradiation technique inside a transmission electron microscope, we show that $\mathrm{ZnO} \mathrm{NWs}$ have remarkable radiation tolerance, manifested by the existence of radiation immune NWs with small diameters, due to the formation of DDZs even at room temperature. Kinetic rate modeling was performed to understand the size dependent migration of defect clusters and variation of defect density in existence of abundant free surfaces. Our study suggests that radiation tolerance of functional oxide NWs has prominent size dependence and the NWs with certain diameters can be potentially immune to radiation, assist their applications in electronic devices in extreme radiation environment.

\section{Methodologies}

\subsection{Materials synthesis and in situ Kr ion irradiation}

$\mathrm{ZnO}$ nanowires were synthesized from $\mathrm{Zn}$ powders (Fisher Scientific, purity $99.3 \%$ ). Before heating up, the powders were placed in a quartz tube furnace, in which $\sim 50$ sccm oxygen flowed for one minute in order to remove air and other residues. Subsequently, the furnace was heated up to $850{ }^{\circ} \mathrm{C}$ at a rate of $\sim 85{ }^{\circ} \mathrm{C} / \mathrm{min}$ and kept for $30 \mathrm{~min}$ with a continuous $\sim 5 \mathrm{sccm} \mathrm{O}_{2}$ 
flow throughout the entire synthesis process. At the end of the annealing, the furnace was naturally cooled at a rate of approximately $10{ }^{\circ} \mathrm{C} / \mathrm{min}$ above $\sim 200{ }^{\circ} \mathrm{C}$ and approximately 1 ${ }^{\circ} \mathrm{C} / \mathrm{min}$ below $\sim 200{ }^{\circ} \mathrm{C}$ [28]. $\mathrm{ZnO} \mathrm{NWs}$ were lifted directly onto carbon-coated grids for transmission electron microscopy (TEM) studies. The microstructure of as-synthesized $\mathrm{ZnO}$ NWs was examined with a Quanta 600 field emission scanning electron microscope and JOEL 2010 transmission electron microscope.

In situ $\mathrm{Kr}^{++}$ion irradiation at an energy of $1 \mathrm{MeV}$ was performed for $\mathrm{ZnO} \mathrm{NWs}$ at room temperature in the Intermediate Voltage Electron Microscope (IVEM) at Argonne National Laboratory, where an ion accelerator was attached to a HITACHI H-9000NAR microscope [29]. The microscope was operated at $200 \mathrm{keV}$ and kept on during radiation in order to record the microstructural evolution. The average dose rate was $2 \times 10^{-3} \mathrm{dpa} / \mathrm{s}$. A CCD camera was used to record the microstructure evolution during ion irradiation at a rate of 15 frames/s. The stopping and range of ions in matter (SRIM) [30] computer program was used to estimate the damage level under ion irradiation. The displacement energies of $\mathrm{Zn}(25 \mathrm{eV})$ and $\mathrm{O}(28 \mathrm{eV})$ were used in the calculation.

\subsection{Rate theory modeling}

The rate theory model is a rather standard model of defect evolution in irradiated materials [31-33]. Our model assumes that the production of interstitials (I) and vacancies (V) are at rates similar to those expected in the experiment, as described below. These defects can diffuse and either annihilate through an $\mathrm{I}+\mathrm{V} \rightarrow 0$ reaction or interstitials can aggregate to form loops $(\mathrm{I}+\mathrm{I} \rightarrow \mathrm{L})$ or interact with existing loops to cause them to grow. Di-interstitial clusters are assumed to be the nucleus of interstitial loops. The surfaces of the wire are assumed to be perfect 
sinks for defects such that all defect concentrations at the surfaces are identically zero. Even though $\mathrm{ZnO}$ is a binary compound and thus there are two types of interstitials and two types of vacancies, the rate theory model only accounts for one type of each species, which is reasonable as the two types of defects behave similarly, especially as predicted by empirical potentials. The expressions governing the evolution of defects in the material are [34]:

$$
\begin{aligned}
\frac{\partial C_{i}}{\partial t} & =D_{i} \frac{\partial^{2} C_{i}}{\partial x^{2}}+P\left(1-C_{v}\right)\left(1-Z C_{v}\right)-R_{i v} C_{i} C_{v}-2 R_{i i} C_{i} C_{i}-\Theta(\alpha) Z D_{i} \sqrt{ } C_{i L} C_{L} C_{i} \\
\frac{\partial C_{v}}{\partial t} & =D_{v} \frac{\partial^{2} C_{v}}{\partial x^{2}}+P\left(1-C_{v}\right)\left(1-Z C_{v}\right)-R_{i v} C_{i} C_{v}-Z D_{v} \sqrt{ } C_{i L} C_{L} C_{v} \\
\frac{\partial C_{L}}{\partial t} & =R_{i i} C_{i} C_{i} \\
\frac{\partial C_{i L}}{\partial t} & =\Theta(\alpha) Z D_{i} \sqrt{C_{i L} C_{L}} C_{i}-Z D_{v} \sqrt{C_{i L} C_{L}} C_{v}+A_{i L} \frac{\partial C_{L}}{\partial t}
\end{aligned}
$$

Physical meanings of the variables in the above equations were listed in Table 1. The first two equations represent the time evolution of the concentration of interstitials and vacancies, respectively, the third equation is for the concentration of interstitial loops, and the fourth is for the concentration of interstitials in loops. All concentrations are a function of time and distance (x) across the wire. As mentioned, we explicitly consider only one type of interstitial and vacancy defect. Other parameters defining the model include $R_{i v}$ and $R_{i i}$, which are the reaction coefficients for interstitials with vacancies and interstitials with interstitials, and are defined as $R_{i v}=Z\left(D_{i}+D_{v}\right), R_{i i}=Z\left(D_{i}+D_{i}\right) . Z$ is the recombination volume, taken to be 24 a [34], with a $=0.321$ $\mathrm{nm}$, and $D$ is the diffusivity of each defect defined by [35]

$$
D={ }_{6}^{1} a^{2} v_{0} e^{-E_{B} T}
$$

where $v_{0}=10 \%$ is the rate prefactor, $E_{m}$ is the migration energy, $T$ is the temperature, and $k_{B}$ is the Boltzmann constant. The migration energy of interstitials, $E_{m}$, is the primary quantity varied in our simulations. The average loop size at any given time and position, measured as the 
average number of interstitials per loop, is defined as $\left\langle N_{i}\right\rangle=C_{i L} / C_{L} . \alpha$ is then defined as $N_{\text {max }}{ }^{-}$ $<N_{i}>$ and thus $\Theta(\alpha)$, where $\Theta$ is the step function, turns off the growth of loops due to the arrival of interstitials for loops containing $N_{\max }$ or more interstitials. In one scenario, $N_{\max }$ is effectively set to infinity, while in the second scenario, labeled as "arrested," interstitial interaction with the existing loops is suppressed in regions of the wire where the average loop size exceeds a predefined value, estimated from the experiments. This maximum (arrest) value is estimated from the fact that the largest loop size in the experiments is roughly $6 \mathrm{~nm}$ in diameter. If one assumes that all of the interstitials in the loop are in one plane, this corresponds to roughly $N_{\max }=600$ atoms. Finally, $P$ is determined from the damage rate estimated by SRIM calculations. The estimated damage rate is $2 \times 10^{-3} \mathrm{dpa} / \mathrm{s}$. This is the number of displacements per atom per second in the material. Given the density of $\mathrm{ZnO}$ is 83.9 atoms $/ \mathrm{nm}^{3}$, this gives a displacement rate of $1.678 \times 10^{-1}$ displacements $/ \mathrm{nm}^{3} \mathrm{~s}$. This final value is converted to actual defects produced by recognizing that not all displacements lead to a defect. After Katoh et al.[33], we use a factor of about 0.2 to convert displacements to damage, giving a final value for $P=0.03356$ defects $/ \mathrm{nm}^{3} \mathrm{~s}$. Rate theory modeling will be applied to investigate size dependent radiation damage in $\mathrm{ZnO}$ nanowires in this study.

\section{Results}

\subsection{Experimental}

As-received ZnO NWs had a hexagonal shaped cross section as shown in Fig. 1a. TEM micrograph and selected area diffraction (SAD) pattern in Fig. 1b-c revealed that a majority of these NWs were single crystals with Wurtzite phase. ZnO NWs with different diameters (30-250 $\mathrm{nm})$ were subjected to $\mathrm{Kr}$ ion irradiation at room temperature to a dose of 5 displacements-per- 
atom (dpa). Radiation-induced damage was examined by TEM using two-beam conditions with diffraction vector $g=[\overline{12}]$. It is evident in Fig. 2 that a large number of dislocation loops were generated after radiation of a NW with an average diameter (D) of $250 \mathrm{~nm}$. Furthermore DDZs with a width of $\sim 25 \mathrm{~nm}$ were observed near surface of the NW. Dislocation-loop DZs were also apparent in irradiated ZnO NWs with an average D ranging from 70 to $100 \mathrm{~nm}$ (Fig. 2b-c). When $\mathrm{D}=30 \mathrm{~nm}$ (Fig. 2d), no dislocation loops were observed, NWs of such small diameters were nearly free of observable radiation-induced defects. The high resolution TEM (HRTEM) image in Fig. 2e shows some typical dislocation loops located on $\{0001\}$ planes in irradiated $\mathrm{ZnO} \mathrm{NW}$ ( $\mathrm{D}=70 \mathrm{~nm}$ ). In comparison, the HRTEM image of irradiated $30 \mathrm{~nm} \mathrm{ZnO}$ NWs in Fig. 2f shows a nearly perfect NW with no evidence of dislocation loops.

Statistical analysis on distribution of dislocation loop density across the NWs (in Fig. 3) shows interesting parabolic trends for NWs with D of $70-250 \mathrm{~nm}$. The peak density of dislocation loops occurs in the center of the wires, and the loop density in $30 \mathrm{~nm} \mathrm{ZnO} \mathrm{NW}$ is essentially zero. The peak density of dislocation loops scales linearly with D as shown in Fig. $3 b$. Meanwhile the distribution of dislocation loop size in irradiated NWs with different diameters (in Fig. 4) shows that by reducing D from 250 to $70 \mathrm{~nm}$, the average dimension of defect clusters $\mathrm{d}_{\text {loop }}$ decreased from $\sim 6$ to $\sim 4 \mathrm{~nm}$. Furthermore radiation-induced dislocation segments with an average length of $18 \mathrm{~nm}$ were observed, while they were absent in NWs when $\mathrm{D}=70 \mathrm{~nm}$.

Radiation-induced swelling and the interaction between preexisting prismatic loops (L1L3 in Fig. 5a) and radiation-induced loops were analyzed. Under irradiation, these preexisting large dislocation loops migrated along the prismatic plane of the NW at a velocity of $\sim 2 \mathrm{~nm} / \mathrm{s}$ in the early stage and slowed down to be eventually stationary (see supplementary video). By 1.5 dpa, as shown in Fig. 5b, these preexisting dislocation loops were pinned by the high-density 
radiation-induced small dislocation loops. Swelling of the $\mathrm{ZnO} \mathrm{NWs}$ along the radial direction was observed during irradiation. For instance the diameter of a wire that was initially $168 \mathrm{~nm}$ (Fig. 5a) increased to $180 \mathrm{~nm}$ by $1.5 \mathrm{dpa}$ (Fig. 5b). Radiation-induced radial strain ( $\varepsilon$ ) was calculated by using $\varepsilon=\left(d-d_{0}\right) / d_{0}$, where $d_{0}$ and $d$ are the wire diameter before and after radiation. Comparison of the variation of radial strain for various NWs in Fig. 5c shows that (1) the radial strain increased monotonically for wires with $\mathrm{D}$ of $\sim 60 \mathrm{~nm}$ or greater; (2) the magnitude of strain decreased for wires of smaller diameter subjected to the same magnitude of radiation dose; (3) the rate of increase in strain during the early stage of irradiation is also lower for wires of smaller diameter. Radial strain was not detectable for wires with $\mathrm{D}$ of $30 \mathrm{~nm}$ or smaller. A magnified region of Fig.5b, shown in Fig. 5d, illustrates that a high density of small radiation-induced dislocation loops were trapped by the preexisting large dislocation loops, leading to the formation of dumbbell-shape distribution of defect clusters, accompanied by the surface DZs with width of $25 \mathrm{~nm}$ or less. Fig. 6 illustrates the uniform DDZs in the regions without pristine dislocation loops (in Fig. 6a) and dumbbell-shape distribution of defect clusters due to the attraction of preexisting dislocation loops (in Fig. 6b).

\subsection{Modeling}

Vacancy migration is generally slow in $\mathrm{ZnO}$ with migration energy in excess of $1 \mathrm{eV}$. The values of the migration energy of interstitials in $\mathrm{ZnO}$ vary significantly $(0.1-0.6 \mathrm{eV})$ in the literature [36, 37]. Values from empirical potentials [37] tend to be higher than those derived using density functional theory (DFT) $[36,38]$, which are sensitive to the charge state. Here a two-dimensional rate theory model was used to understand size dependent defect evolution in irradiated ZnO NWs. Fig. 7a shows the crystallographic structure of defect in our modeling 
studies: a di-interstitial cluster (I2) in Wurtzite-structured ZnO. Fig. 7b compares the simulated loop density profiles across the wire as a function of NW diameter when the migration energy of interstitials $\mathrm{E}_{\mathrm{m}}=0.15 \mathrm{eV}$. These simulated profiles are similar to experimental observations in that they clearly show a parabolic distribution of loop density and smaller wires lead to much fewer dislocation loops. Furthermore, limiting the maximum size of loops (indicated as "arrested" for $100 \mathrm{~nm} \mathrm{NW)} \mathrm{also} \mathrm{increases} \mathrm{the} \mathrm{loop} \mathrm{density} \mathrm{(comparing} \mathrm{to} \mathrm{unarrested} \mathrm{state)} \mathrm{as} \mathrm{more}$ interstitials are free to nucleate loops. However, as Fig. 8a reveals, as $\mathrm{E}_{\mathrm{m}}$ is increased beyond $0.15 \mathrm{eV}$, the dependence of loop density on wire diameter diminishes. When $\mathrm{E}_{\mathrm{m}}$ is unrealistically large, $\geq 0.4 \mathrm{eV}$, all wires exhibit similar loop densities in the center of wires. Meanwhile the simulated loop density increases with greater $\mathrm{E}_{\mathrm{m}}$ because interstitials cannot diffuse very far and loop nucleation is dominated not by interstitial diffusion to existing loops but by the creation of a high concentration of interstitials during the damage events such that the clustering probability of less mobile interstitials significantly increases. Fig. $8 \mathrm{~b}$ illustrates that as the migration energy of interstitials increases, the average loop size in the center of the wires decreases. That is, as the number of loops increases, the average size decreases. Conversely, when the migration energy is small, the average loop size predicted by the model becomes quite large, almost $100 \mathrm{~nm}$ in diameter, unless the maximum size of interstitial loops is limited (as indicated by "arrested") to be comparable to the experimental observations. In $\mathrm{MgAl}_{2} \mathrm{O}_{4}$, interstitial loops are found to grow only to a maximum size as they are faulted loops and the fault energy results in an energetic penalty for further growth [39].

To assess the sensitivity of the predicted results on our model, we considered two other variants of the model. Simulations using empirical potentials show that interstitial clusters in $\mathrm{ZnO}$ can have very high mobility, just as we found in $\mathrm{MgO}$ [40]. We thus constructed a model in 
which we explicitly tracked di-interstitial clusters and considered a loop to be nucleated with clusters of sizes greater than 2 formed. The basic qualitative behavior did not change, as the loop distributions were governed by the slower moving species. That is, if clusters diffuse much quicker than mono-interstitials, they quickly find mono-interstitials (as at these radiation conditions there is always a large number of interstitials being produced) and nucleate a loop.

The second variant explicitly tracked $\mathrm{Zn}$ defects separately from $\mathrm{O}$ defects. In this model, the mobility of each type of interstitial could be changed independently, in accordance with theoretical results[36-38]. Again, as for the cluster model above, the behavior was dominated by the slower moving species. The results from this two-interstitial model and the original oneinterstitial model are very similar if the mobility of the slowest interstitial was the same in both cases. That is, the behavior of this two-interstitial model can be captured in the one-interstitial model by setting the migration energy of the single interstitial to that of the slower interstitial in the two-interstitial model. We thus conclude that these variants, while maybe slightly changing the predicted loop densities and distributions, do not change the qualitative behavior, at least for the range of mobilities considered here.

\section{Discussion}

\subsection{Defect denuded zones (DDZs) and swelling in NWs}

The formation of DDZs in ZnO NWs is the result of the abundant defect sinks, free surface in particular. The width of the DDZs is strongly coupled to the migration energy ( $\left.E_{m}\right)$ of interstitials. Our modeling shows that, for there to be a strong size (wire diameter) dependent loop density at room temperature, interstitials must diffuse quickly with a low $\mathrm{E}_{\mathrm{m}}(\sim 0.15 \mathrm{eV})$. Slow migration leads to essentially homogeneous loop distributions across the wire. The small value of $\mathrm{E}_{\mathrm{m}}$ is in 
agreement with DFT calculations[36]. However, the absolute magnitude of loop densities simulated in our model is smaller than the experimental observations. A number of factors may contribute to this disparity. First, in situ studies show that the preexisting loops trap radiationinduced loops and balance the sink effect from free surface, resulting in drastic increase of loop density locally. Second, electrostatic interactions between interstitials and loops may be significantly weaker than those between two interstitials, increasing the rate of $\mathrm{I}+\mathrm{I} \rightarrow \mathrm{L}$ loop reactions over $\mathrm{I}+\mathrm{L}$ reactions (where $\mathrm{L}$ stands for loop), which is not accounted for in the model. Third, the rate of annihilation $(\mathrm{I}+\mathrm{V} \rightarrow 0)$ recombinations might also be reduced if vacancies are allowed to form small clusters that do not interact with interstitials as strongly via electrostatics as monovacancies do. Finally, the actual Frenkel pair production rate may be higher in the experiments than estimated in the model or loops may essentially nucleate within the cascade. Both of these would tend to increase the loop density as interstitials and loop nuclei would be produced in higher quantities. Accounting for all of these factors that would lead to full agreement between the model and experiment is challenging, but the qualitative agreement reveals that both the dependence of the loop density on wire diameter and the DDZs observed near the surface of the wires is a direct consequence of the high mobility of interstitials.

Swelling of $\mathrm{ZnO} \mathrm{NWs}$ along the radial direction observed under irradiation at room temperature mainly originates from the formation of interstitials clusters. The swelling rate of NW with diameter of $168 \mathrm{~nm}$ (Fig. 5c) has probably undergone an initial primary stage at high swelling rate, followed by a secondary stage at much lower rate. In comparison, as the wire diameter decreased, the primary swelling rate declined, implying an enhanced swelling resistance in wires of smaller diameters. Alleviation of swelling in $\mathrm{ZnO} \mathrm{NWs}$ of smaller 
diameters is a direct consequence of size dependent reduction of defect density due to the existence of free surface.

\subsection{Dumbbell-shape distribution of dislocation loops in NWs}

Free surface has hitherto known to be the strongest defect sinks. Without the influence of pristine loops, a uniform defect DDZ typically forms in ZnO NWs with a width of $\sim 25 \mathrm{~nm}$, as illustrated in Fig. 2a-c. However, in presence of pristine loops, we observed the dumbbell-shape distribution of dislocation loops (Fig. 5d). Such a surprising observation indicates that the stress field from these large loops (with size comparable to wire diameter) counteracts the image forces arising from image dislocations in close proximity to the free surfaces (as shown schematically in Fig. 6b) and fetters the radiation-induced small loops from escaping to the free surface.

Next we attempt to compare the stress field from the large loops and free surface. Khraishi et al. [41] calculated the stress field of a circular Volterra dislocation loop normal to the plane of a loop. Using the geometry of a pristine loop, shown schematically in Fig. 9a-b, we calculate the relevant stress field components $\left(\sigma_{x x}\right.$ and $\left.\sigma_{y y}\right)$ of the large pristine loop by using:

$$
\begin{aligned}
\sigma_{x x} & =\frac{x^{2}}{\rho^{2}}\left(-\frac{G b_{z}}{\pi}\left[C_{1} \mathbf{E}(k)+C_{2} \mathbf{K}(k)\right]-\frac{G b_{z}}{2 \pi(1-v)}\left[C_{3} \mathbf{E}(k)+C_{4} \mathbf{K}(k)\right]\right) \\
& +\frac{y^{2}}{\rho^{2}}\left(-\frac{G b_{z}}{\pi}\left[C_{5} \mathbf{E}(k)+C_{6} \mathbf{K}(k)\right]-\frac{G b_{z}}{2 \pi(1-v)}\left[C_{7} \mathbf{E}(k)+C_{8} \mathbf{K}(k)\right]\right)
\end{aligned}
$$

where the shear modulus $G=44 \mathrm{GPa}$, and Poisson's ratio $v=0.35 . \mathbf{K}(\mathrm{k})$ and $\mathbf{E}(\mathrm{k})$ are complete elliptic integrals of the first and second kinds respectively, where $\mathrm{k}$ is the modulus of these integrals or functions. $b_{z}=0.520 \mathrm{~nm}$ is the magnitude of Burgers vector along z-axis. Coefficients $\mathrm{C}_{1}$ to $\mathrm{C}_{8}$ are related to the spatial coordinates $(\mathrm{x}, \mathrm{y}, \mathrm{z})$ and the loop radius, $\mathrm{R}$. The resultant of stresses, $\sigma_{x x}^{\text {loop }}$ and $\sigma_{y y}^{\text {loop }}$, are calculated as a function of distance from the preexisting 
large loops. It is evident that the magnitude of the stress decreases at locations farther away from the center of the two preexisting large loops. Meanwhile the free surface imposes two competing image stresses $\sigma_{x x}^{\text {image }}$ and $\sigma_{y y}^{\text {image }}$, calculated by using similar method for dislocation loops with a diameter of $6 \mathrm{~nm}$. Comparisons of the stress field resulted from the large loops and the image force are illustrated in Fig. 9c. The image stress imposed on the small loops in the middle plane $(\mathrm{z}=0)$ at $25 \mathrm{~nm}$ from the free surface is calculated to be $\sim 35 \mathrm{MPa}$, which is nearly identical to $\sigma_{x x}^{\text {loop }}$ and $\sigma_{y y}^{\text {loop }}$ arising from the pristine large loops. Within the dumbbell region the pristine loops exert more stress on the small loops, whereas the image stress prevails in the regions outside the dumbbell region. Foregoing discussions confirm that the attractive stress field from the pristine large loops can compete with the image stress or prevail in certain cases, leading to the observation of high density of dislocation loops in irradiated large NWs.

\section{Conclusions}

In situ $\mathrm{Kr}$ ion irradiations of $\mathrm{ZnO} \mathrm{NWs}$ at room temperature show the existence of DDZs with a width of $\sim 25 \mathrm{~nm}$ near free surfaces. The parabolic distribution of defect density in the wires, the size-dependent reduction of defect density and swelling are due to the surface defect sinks and low interstitial migration energy $(\sim 0.15 \mathrm{eV})$. NWs with sufficiently small wire diameters are nearly immune to radiation, manifested by the absence of interstitial loops and diminished radiation-induced swelling and creep. Preexisting dislocation loops show surprisingly large drag force that can compete with free surface. This study implies that nanowires of certain diameters may significantly enhance the radiation tolerance of oxides in general for device applications in extreme radiation environment. 


\section{Acknowledgements}

We acknowledge financial support by NSF-DMR-Metallic Materials and Nanostructures Program under grant no. 1304101. C. S. was supported by DOE-NEUP under contract no. DEAC07-05ID14517-00088120. B. P. U. acknowledges support by the U.S. Department of Energy,

Office of Science, Basic Energy Sciences, Materials Sciences and Engineering Division. Los Alamos National Laboratory, an affirmative action equal opportunity employer, is operated by Los Alamos National Security, LLC, for the National Nuclear Security Administration of the U.S. DOE under contract DE-AC52-06NA25396. The IVEM facility at Argonne National Laboratory is supported by DOE-Office of Nuclear Energy. 


\section{References}

[1] K. Murty, I. Charit. Structural materials for Gen-IV nuclear reactors: challenges and opportunities, J. Nucl. Mater. 383 (2008) 189-195.

[2] W.J. Weber, R.C. Ewing, C. Catlow, T.D.d.l. Rubia, L. Hobbs, C. Kinoshita, H. Matzke, A. Motta, M. Nastasi, E. Salje. Radiation effects in crystalline ceramics for the immobilization of high-level nuclear waste and plutonium, J. Mater. Res. 13 (1998) 1434-1484.

[3] L.W. Hobbs, F.W. Clinard Jr, S.J. Zinkle, R.C. Ewing. Radiation effects in ceramics, J. Nucl. Mater. 216 (1994) 291-321.

[4] S. Zinkle. Fundamental radiation effects parameters in metals and ceramics, Radiat Eff. Defects Solids 148 (1999) 447-477.

[5] S.J. Zinkle. Effect of irradiation spectrum on the microstructural evolution in ceramic insulators, J. Nucl. Mater. 219 (1995) 113-127.

[6] F.W. Clinard Jr, G.F. Hurley, L.W. Hobbs. Neutron irradiation damage in $\mathrm{MgO}, \mathrm{Al}_{2} \mathrm{O}_{3}$ and $\mathrm{MgAl}_{2} \mathrm{O}_{4}$ ceramics, J. Nucl. Mater. 108-109 (1982) 655-670.

[7] S. Kondo, Y. Katoh, L.L. Snead. Analysis of grain boundary sinks and interstitial diffusion in neutron-irradiated SiC, Phys. Rev. B 83 (2011) 075202.

[8] W. Weber, R. Eby, R. Ewing. Accumulation of structural defects in ion-irradiated $\mathrm{Ca}_{2} \mathrm{Nd}_{8}\left(\mathrm{SiO}_{4}\right)_{6} \mathrm{O}_{2}$, J. Mater. Res 6 (1991) 1334.

[9] A. Matsunaga, C. Kinoshita, K. Nakai, Y. Tomokiyo. Radiation-induced amorphization and swelling in ceramics, J. Nucl. Mater. 179-181, Part 1 (1991) 457-460.

[10] M. Wittels, F.A. Sherrill. Radiation Damage in $\mathrm{SiO}_{2}$ Structures, Phys. Rev. Lett. 93 (1954) 1117-1118.

[11] W. Primak. Fast-Neutron-Induced Changes in Quartz and Vitreous Silica, Phys. Rev. Lett. 110 (1958) 1240-1254.

[12] F.W. Clinard Jr, L.W. Hobbs, C.C. Land, D.E. Peterson, D.L. Rohr, R.B. Roof. Alpha decay self-irradiation damage in ${ }^{238} \mathrm{Pu}$-substituted zirconolite, J. Nucl. Mater. 105 (1982) 248256.

[13] J. Zhang, Y.Q. Wang, M. Tang, C. Sun, D.M. Yin, N. Li. Helium irradiation induced micro-swelling and phase separation in pyrochlore Lu2Ti2O7, Nucl. Instr. Meth. Phys. Res. B 342 (2015) 179-183.

[14] W.J. Weber. Radiation-induced defects and amorphization in zircon, J. Mater. Res. 5 (1990) 2687-2697.

[15] C. Sun, M. Song, K.Y. Yu, Y. Chen, M. Kirk, M. Li, H. Wang, X. Zhang. In situ Evidence of Defect Cluster Absorption by Grain Boundaries in Kr Ion Irradiated Nanocrystalline Ni, Metall Mater Trans A 44 (2013) 1966-1974.

[16] K.Y. Yu, Y. Liu, C. Sun, H. Wang, L. Shao, E.G. Fu, X. Zhang. Radiation damage in helium ion irradiated nanocrystalline Fe, J. Nucl. Mater. 425 (2012) 140-146.

[17] T.D. Shen, S. Feng, M. Tang, J.A. Valdez, Y. Wang, K.E. Sickafus. Enhanced radiation tolerance in nanocrystalline $\mathrm{MgGa}_{2} \mathrm{O}_{4}$, Appl. Phys. Lett. 90 (2007) 263115.

[18] G.R. Odette, M.J. Alinger, B.D. Wirth. Recent Developments in Irradiation-Resistant Steels, Annu. Rev. Mater. Res. 38 (2008) 471-503.

[19] M. Song, Y. Wu, D. Chen, X. Wang, C. Sun, K. Yu, Y. Chen, L. Shao, Y. Yang, K. Hartwig. Response of equal channel angular extrusion processed ultrafine-grained T91 steel subjected to high temperature heavy ion irradiation, Acta Mater. 74 (2014) 285-295. 
[20] K.Y. Yu, D. Bufford, C. Sun, Y. Liu, H. Wang, M.A. Kirk, M. Li, X. Zhang. Removal of stacking-fault tetrahedra by twin boundaries in nanotwinned metals, Nat Commun 4 (2013) 1377. [21] K.Y. Yu, C. Sun, Y. Chen, Y. Liu, H. Wang, M.A. Kirk, M. Li, X. Zhang. Superior tolerance of $\mathrm{Ag} / \mathrm{Ni}$ multilayers against $\mathrm{Kr}$ ion irradiation: an in situ study, Philos. Mag. (2013) $1-16$.

[22] E. Bringa, J. Monk, A. Caro, A. Misra, L. Zepeda-Ruiz, M. Duchaineau, F. Abraham, M. Nastasi, S. Picraux, Y. Wang. Are nanoporous materials radiation resistant?, Nano Lett. 12 (2011) 3351-3355.

[23] C. Sun, D. Bufford, Y. Chen, M.A. Kirk, Y.Q. Wang, M. Li, H. Wang, S.A. Maloy, X. Zhang. In situ study of defect migration kinetics in nanoporous Ag with enhanced radiation tolerance, Sci. Rep. 4 (2014) 3737.

[24] R. Liontas, X.W. Gu, E. Fu, Y. Wang, N. Li, N. Mara, J.R. Greer. Effects of Helium Implantation on the Tensile Properties and Microstructure of Ni73P27 Metallic Glass Nanostructures, Nano Lett. 14 (2014) 5176-5183.

[25] Z.L. Wang. Zinc oxide nanostructures: growth, properties and applications, J. Phys.: Condens. Matter 16 (2004) R829.

[26] Z.L. Wang, J. Song. Piezoelectric nanogenerators based on zinc oxide nanowire arrays, Science 312 (2006) 242-246.

[27] P. Yang, H. Yan, S. Mao, R. Russo, J. Johnson, R. Saykally, N. Morris, J. Pham, R. He, H.-J. Choi. Controlled growth of $\mathrm{ZnO}$ nanowires and their optical properties, Adv. Funct. Mater. $12(2002) 323$.

[28] L. Yin, C. Yu. Facilitating $\mathrm{ZnO}$ nanostructure growths by making seeds for self-catalytic reactions, J. Solid State Chem. 187 (2012) 58-63.

[29] R. Birtcher, M. Kirk, K. Furuya, G. Lumpkin, M. Ruault. In situ transmission electron microscopy investigation of radiation effects, J. Mater. Res. 20 (2005) 1654-1683.

[30] J.F. Ziegler, J.P. Biersack, M.D. Ziegler. The Stopping and Range of Ions in Matter, 2008. [31] N. Yoshida, M. Kiritani. Point defect clusters in electron-irradiated gold, J. Phys. Soc. Jpn. 35 (1973) 1418-1429.

[32] M.J. Demkowicz, R.G. Hoagland, B.P. Uberuaga, A. Misra. Influence of interface sink strength on the reduction of radiation-induced defect concentrations and fluxes in materials with large interface area per unit volume, Phys. Rev. B 84 (2011) 104102.

[33] Y. Katoh, T. Muroga. Characterization of cascade-induced defect production by microstructural analysis based on rate theory modeling, J. Nucl. Mater. 233-237, Part 2 (1996) 1029-1034.

[34] G.S. Was. Fundamentals of radiation materials science, Springer, 2007.

[35] D.A. Porter, K.E. Easterling. Phase Transformations in Metals and Alloys, (Revised Reprint), CRC press, 1992.

[36] P. Erhart, K. Albe. Diffusion of zinc vacancies and interstitials in zinc oxide, Appl. Phys. Lett. 88 (2006) 201918.

[37] D. Chen, F. Gao, M. Dong, B. Liu. Migration of point defects and a defect pair in zinc oxide using the dimer method, J. Mater. Res. 27 (2012) 2241-2248.

[38] P. Erhart, K. Albe. First-principles study of migration mechanisms and diffusion of oxygen in zinc oxide, Phys. Rev. B 73 (2006) 115207.

[39] C. Kinoshita, K. Fukumoto, K. Fukuda, F.A. Garner, G.W. Hollenberg. Why is magnesia spinel a radiation-resistant material?, J. Nucl. Mater. 219 (1995) 143-151. 
[40] B. Uberuaga, R. Smith, A. Cleave, F. Montalenti, G. Henkelman, R. Grimes, A. Voter, K. Sickafus. Structure and mobility of defects formed from collision cascades in MgO, Phys. Rev. Lett. 92 (2004) 115505.

[41] T.A. Khraishi, J.P. Hirth, H.M. Zbib, T.D. de La Rubia. The stress field of a general circular Volterra dislocation loop: Analytical and numerical approaches, Philos. Mag. Lett. 80 (2000) 95-105. 
Table 1 List of parameters in the rate theory modeling.

\begin{tabular}{|l|l|l|}
\hline Parameter & Physical Meaning & Value \\
\hline$P$ & Defect production rate & 0.03356 defects $/ \mathrm{nm}^{3} \mathrm{~s}$ \\
\hline$t$ & Simulation time & $2500 \mathrm{~s}$ \\
\hline$T$ & Temperature & $300 \mathrm{~K}$ \\
\hline$a$ & Width of nanowire & variable \\
\hline$v_{0}$ & Nearest neighbor anion-anion distance & $0.321 \mathrm{~nm}$ \\
\hline$E_{m i}$ & Rate prefactor & $10 \% / \mathrm{s}$ \\
\hline$E_{m v}$ & Interstitial migration energy & variable \\
\hline$Z$ & Vacancy migration energy & $1.44 \mathrm{eV}$ \\
\hline$N_{m a x}$ & Reaction volume & $7.704 \mathrm{~nm}$ \\
\hline$A_{i L}$ & Number of interstitials in loop nucleus & 2 interstitials \\
\hline
\end{tabular}



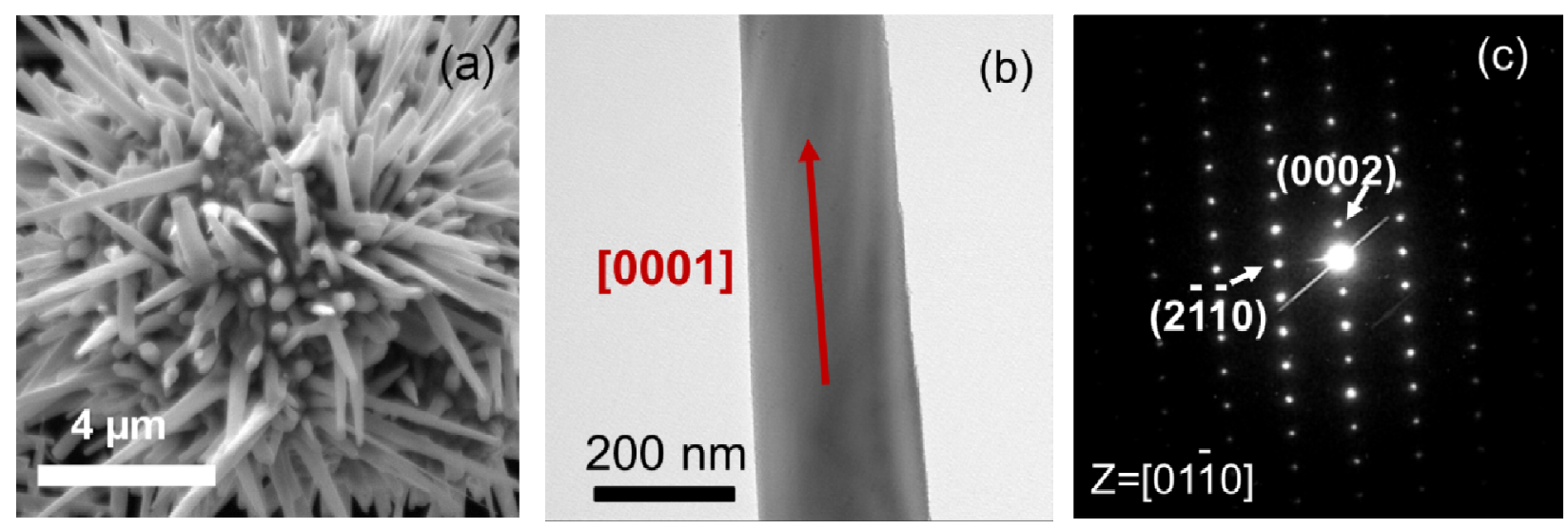

Fig.1. Microstructure of as-fabricated $\mathrm{ZnO}$ nanowires with different diameters. (a) SEM micrograph of as-received $\mathrm{ZnO}$ nanowires. The cross-section of the nanowire shows hexagonal shape. (b) Bright field (BF) TEM micrograph of a typical single crystal $\mathrm{ZnO}$ nanowire grew along <0001> direction. (c) Selected area diffraction (SAD) pattern confirm the formation of single crystal $<0002>\mathrm{ZnO}$ nanowire. 

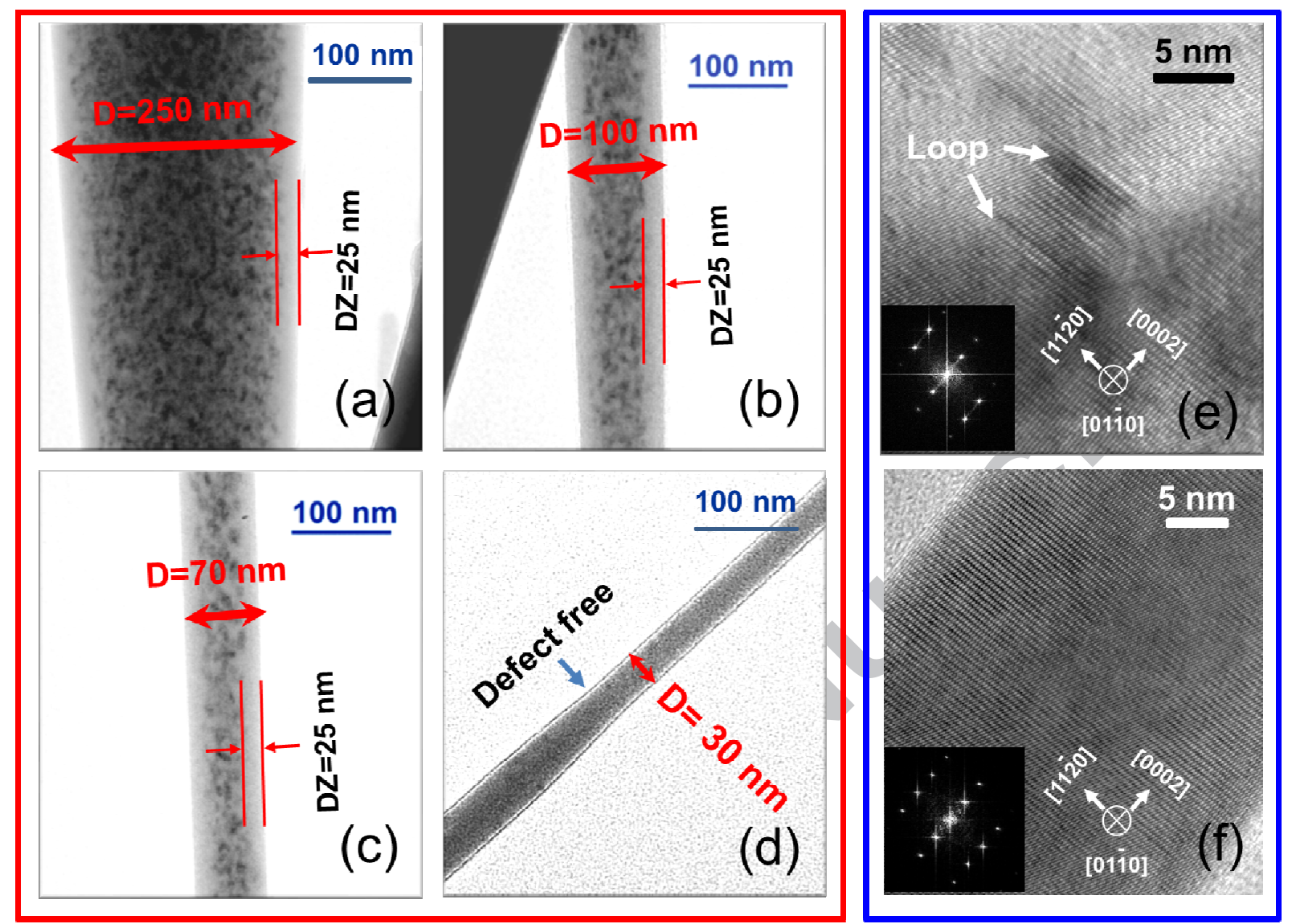

Fig.2. Defect denuded zone (DDZ) in ZnO nanowires with different diameters revealed by in situ $\mathrm{Kr}$ ion irradiations up to $5 \mathrm{dpa}$. (a) TEM micrograph showing a DDZ with a width of $\sim 25 \mathrm{~nm}$ for a $\mathrm{ZnO}$ nanowire with an average diameter $\mathrm{D}$ of $\sim 250 \mathrm{~nm}$, A similar width of DDZ was also displayed in nanowires with D of $100 \mathrm{~nm}$ (b) and $70 \mathrm{~nm}$ (c). Meanwhile the density of defect clusters appeared to reduce with decreasing wire diameter. (d) When D $=30 \mathrm{~nm}$, no clear evidence of dislocation loop can be observed, that is the wire of this diameter is immune to radiation induced damage. (e) High resolution TEM (HRTEM) image showing dislocation loops in irradiated nanowire with $\mathrm{D}$ of $70 \mathrm{~nm}$. The dislocation loops, with $\sim 5 \mathrm{~nm}$ in diameter, were located on $\{0001\}$ plane. (f) HRTEM image of irradiated nanowire $(D=30 \mathrm{~nm})$ showing the absence of dislocation loops. 

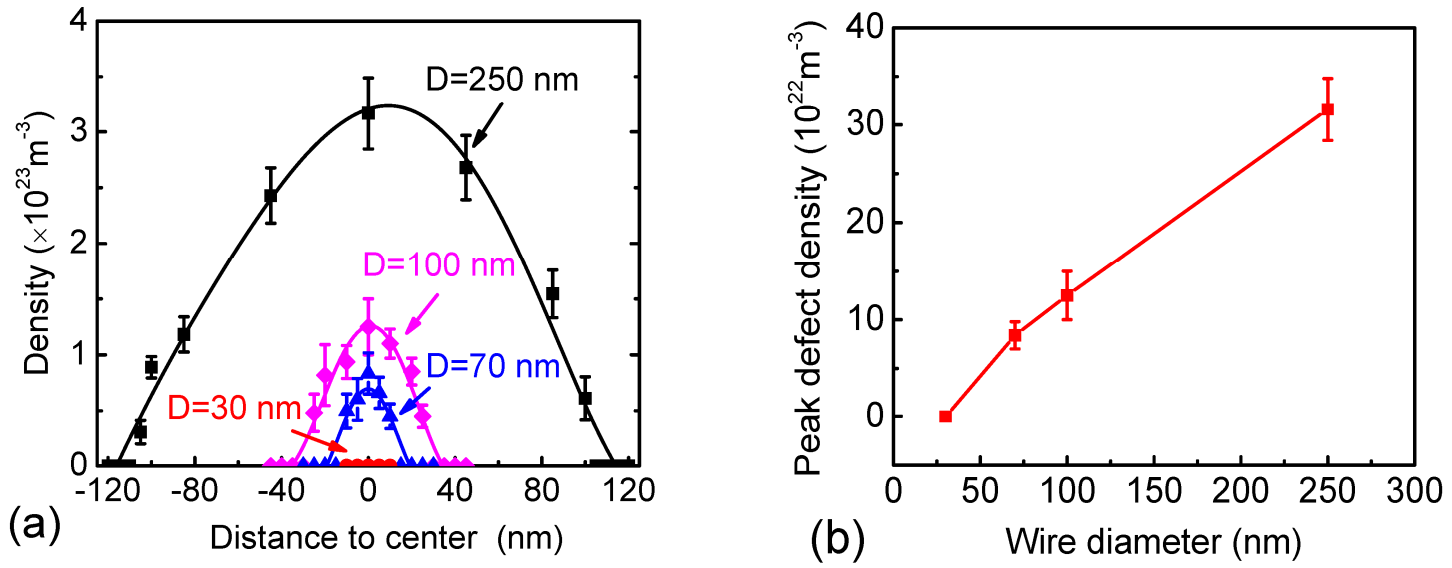

Fig.3. Quantitative analyses of size dependent enhancement of radiation tolerance in $\mathrm{ZnO}$ nanowires. (a) Defect cluster density as a function of distance to the center of wires for $\mathrm{Kr}$ ion irradiated $\mathrm{ZnO}$ nanowires with diameters of 30-250 nm. Parabolic distribution of defect density was observed in most cases, that is, defect density in central region is greater than that close to surfaces. (b) Wire size dependent peak dislocation loop density. The peak defect density is substantially higher in wires of larger diameters. When the wire diameter reduces to $30 \mathrm{~nm}$, the $\mathrm{ZnO}$ nanowire is basically free from visible dislocation loops. 

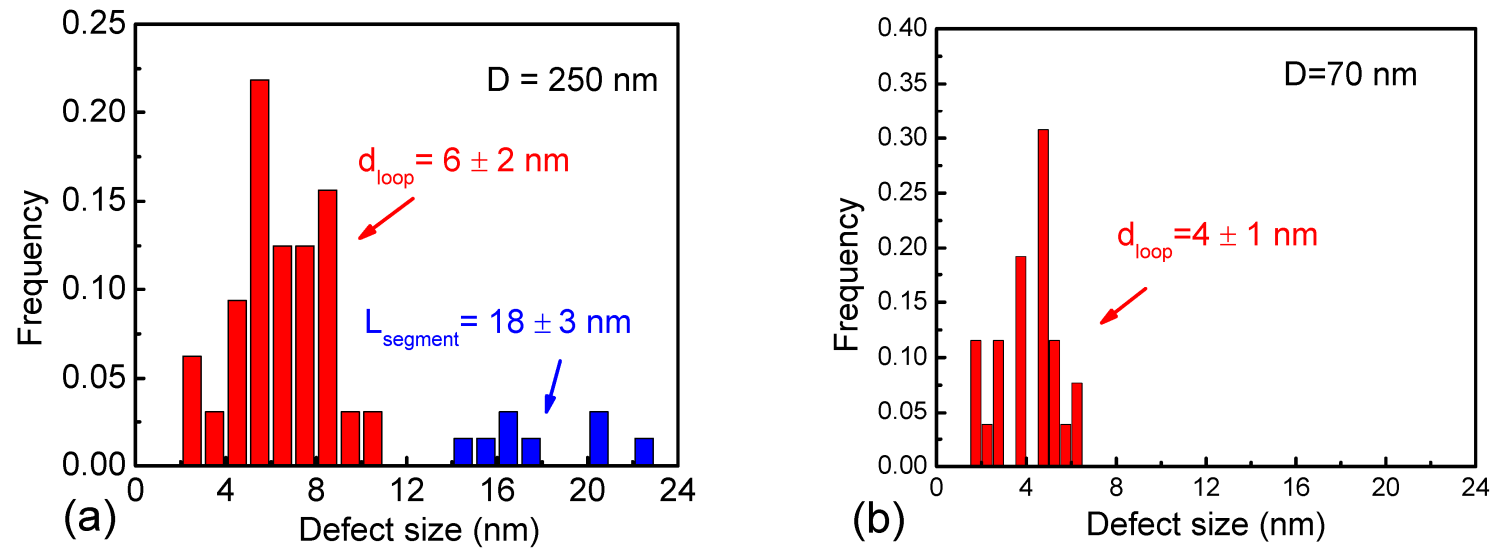

Fig.4. Comparison of statistical distribution of defect size in wires of $250 \mathrm{~nm}$ (a) and $70 \mathrm{~nm}$ (b) in diameter show that the average diameter of dislocation loops $\left(\mathrm{d}_{\text {loop }}\right)$ reduced with decreasing wire diameter. The dislocation segments formed in the wire with $\mathrm{D}$ of $250 \mathrm{~nm}$ were absent in the wire with D of $100 \mathrm{~nm}$ or less. 

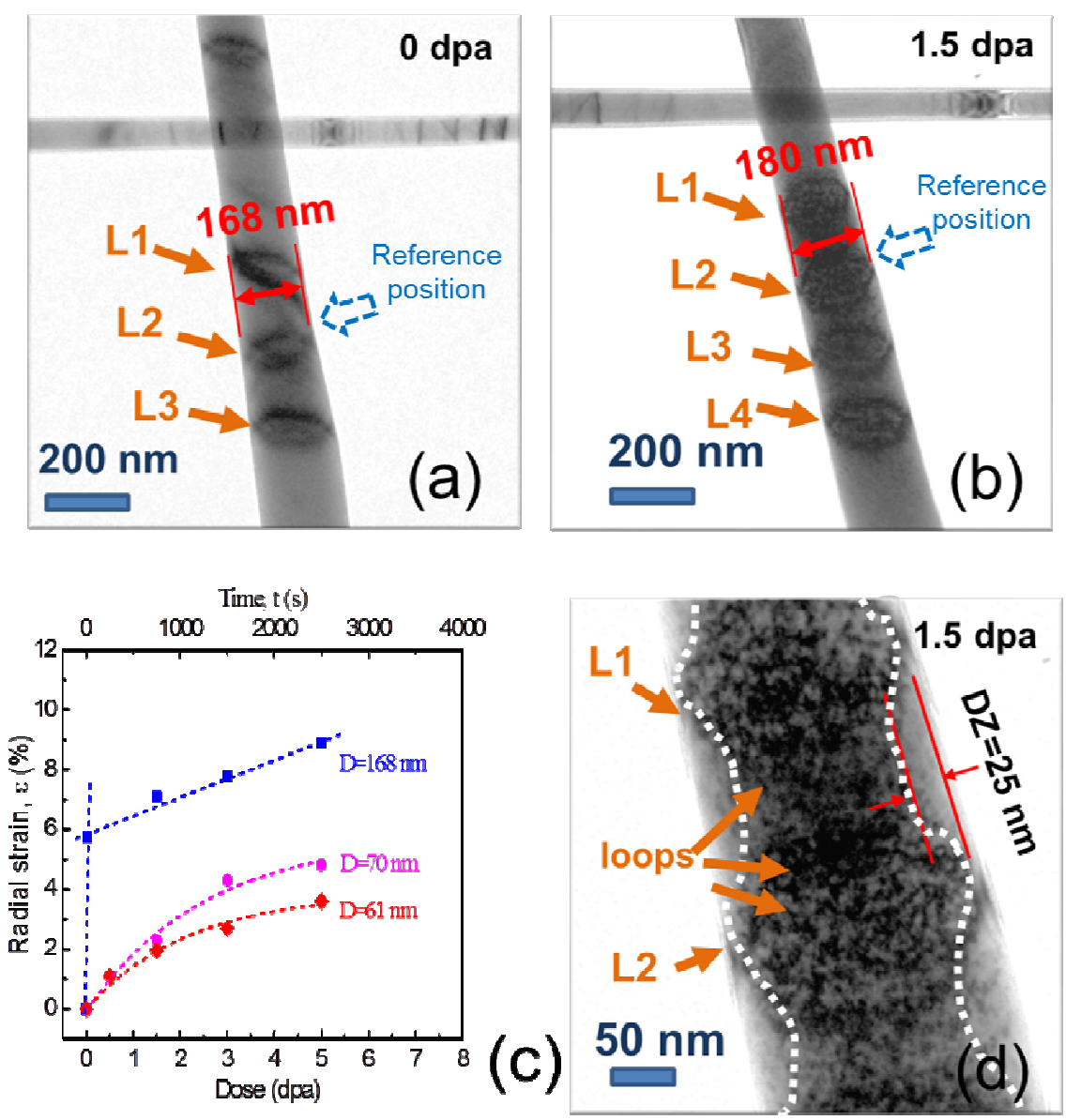

Fig.5. Radiation-induced swelling and interaction of large preexisting pristine dislocation loops with radiation-induced small defect clusters. (a) Bright field TEM image of several preexisting dislocation loops (L1-L3) in $\mathrm{ZnO}$ nanowire prior to irradiation. (b) After $\mathrm{Kr}$ ion irradiation up to $1.5 \mathrm{dpa}$, a high density of defect clusters was observed near an array of hexagonal shaped preexisting dislocation loops (L1- L3). The wire diameter (measured from the same location) swelled from 168 to $180 \mathrm{~nm}$, and the dash arrows show the reference position for the measurements. Notice that another loop (L4) migrated upward along wire axial direction during radiation. (c) Comparison of radiation induced swelling (radial strain) in wires of various diameters shows that wires of larger diameter $(D=168 \mathrm{~nm})$ swelled rapidly to $\sim 9.0 \%$ by 5 dpa. In contrast, wires of smaller initial diameter, $61 \mathrm{~nm}$, swelled gradually and moderately to $\sim 3.6 \%$. (d) Magnified TEM micrograph of the loops L1 and L2 in Fig. 5b shows that a large number of small dislocation loops were trapped by pristine dislocation loops, and consequently a dumbbell distribution of small loops was observed. 


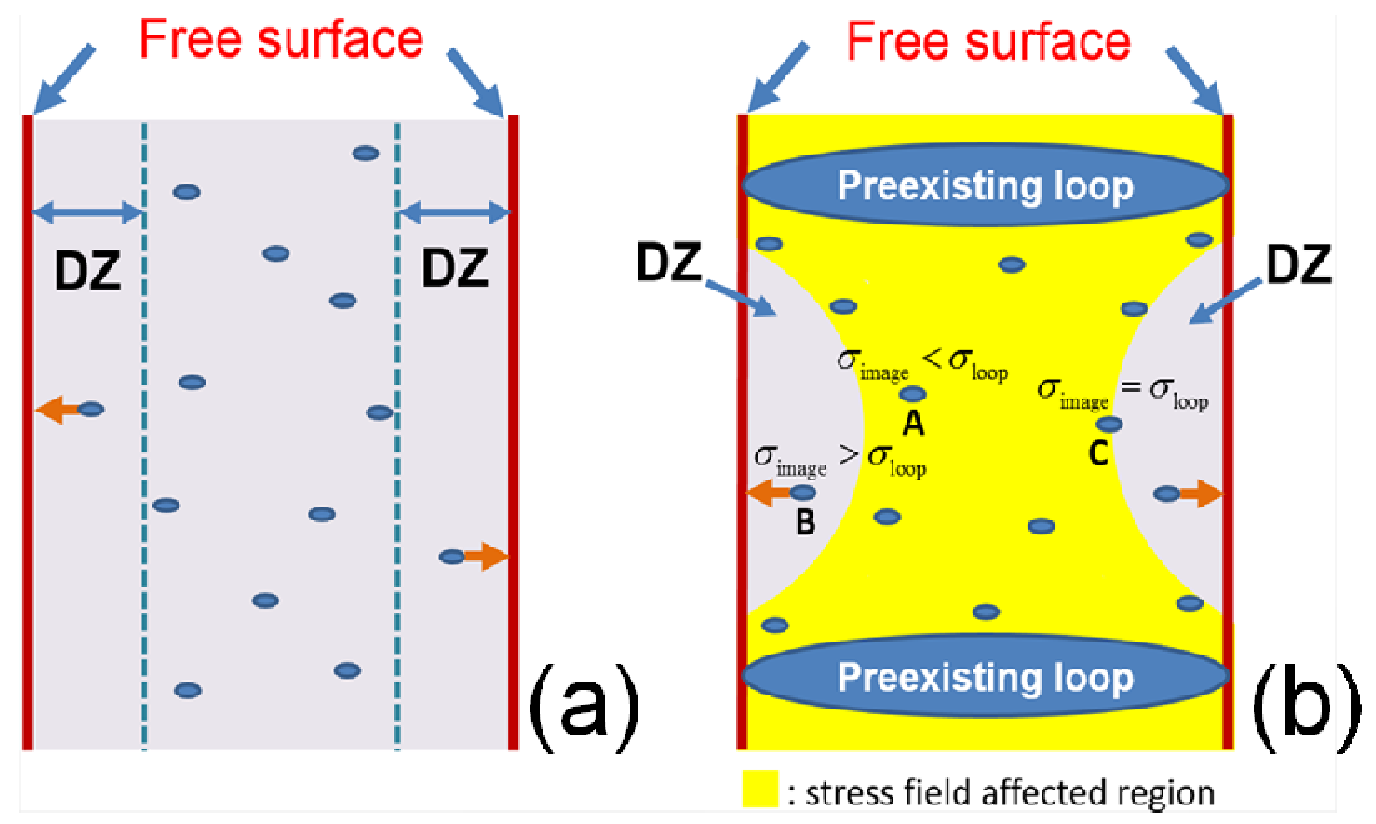

Fig.6. (a) Schematic illustration of uniform DDZs in the region without pristine dislocation loops. (b) With preexisting dislocation loops, the attraction of radiation-induced loops by preexisting loops results in the dumbbell-shape distribution of defect clusters and non-uniform DDZs. 
(a)
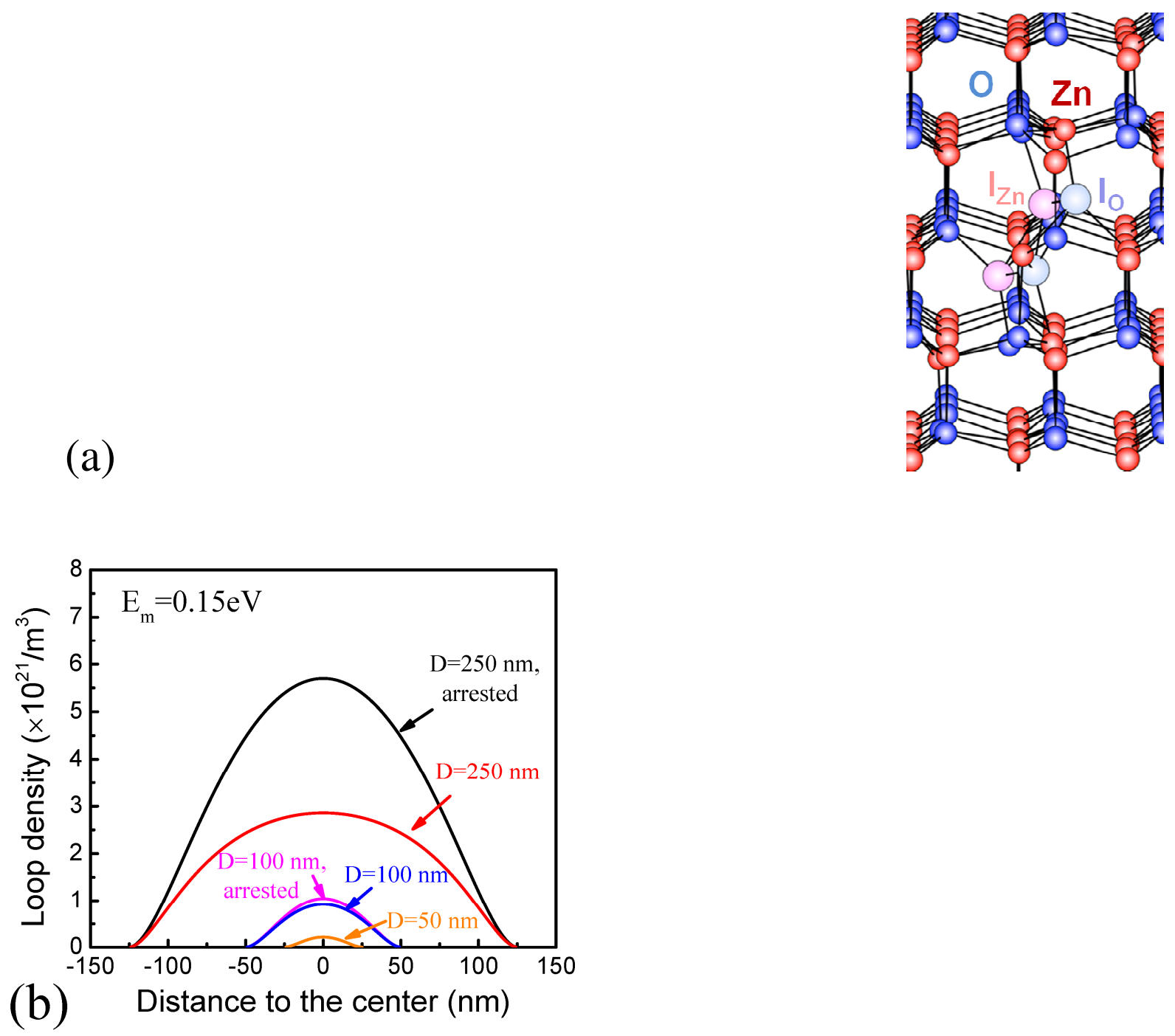

Fig.7. (a) Structure of the di-interstitial cluster (I2) in wurtzite-structured $\mathrm{ZnO}$. The interstitial atoms are highlighted (light colored larger spheres). Essentially, this structure has two split interstitials, one $\mathrm{O}$ and one $\mathrm{Zn}$, next to each other. (b) Distribution of dislocation loop density vs. the distance to the center of the wires of various diameters. The interstitial migration energy $\mathrm{E}_{\mathrm{m}}$ is $0.15 \mathrm{eV}$. "Arrested" indicates that the maximum loop size was limited. 


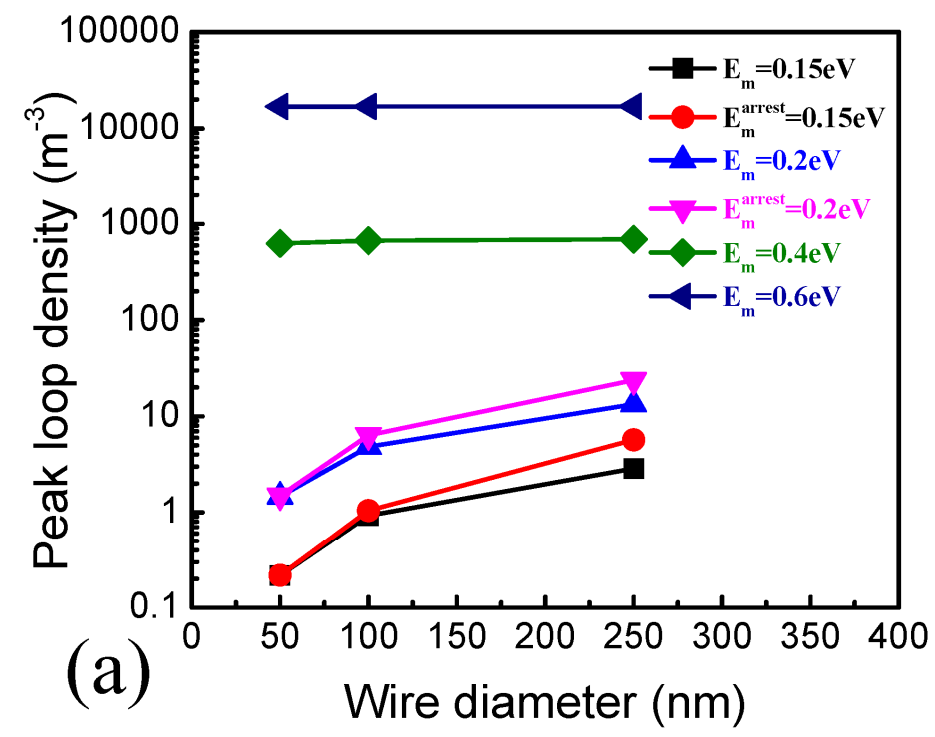




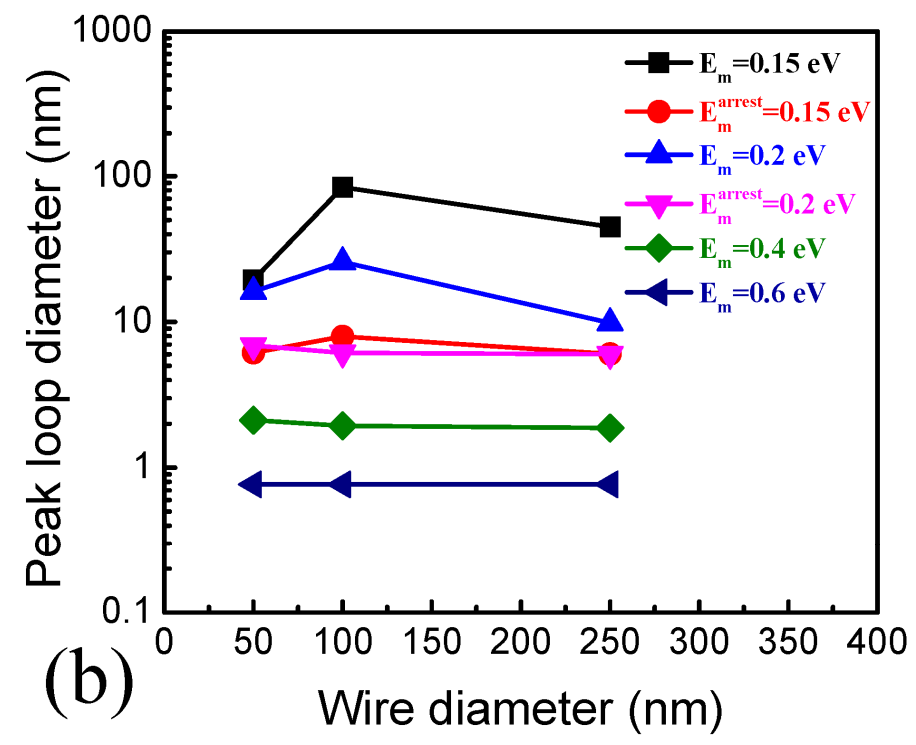

Fig.8. (a) Loop density in the center of the wire as a function of wire diameter and interstitial migration energy $\left(E_{m}\right)$. When $E_{m}$ is in the range of $0.15-0.2 \mathrm{eV}$, a clear size dependent reduction of defect density is visible. (b) The average loop size in the center of the wire varies as a function of $\mathrm{E}_{\mathrm{m}}$, and in general depends little on wire diameter. When $\mathrm{E}_{\mathrm{m}}^{\text {arrest }}=0.15-0.2 \mathrm{eV}$, the estimated loop diameter is consistent with experimental studies. 


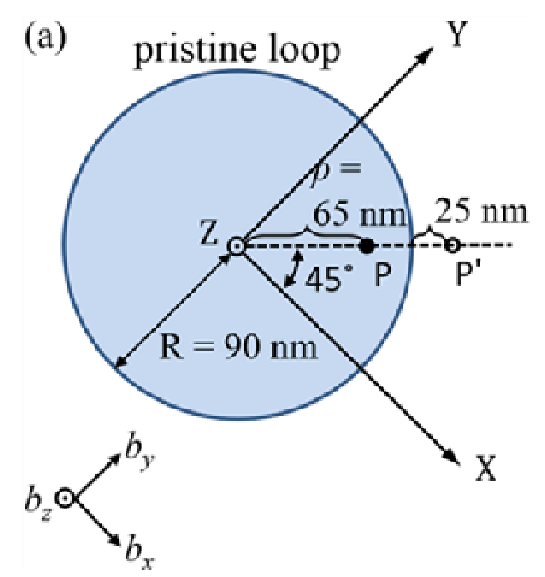

(c)
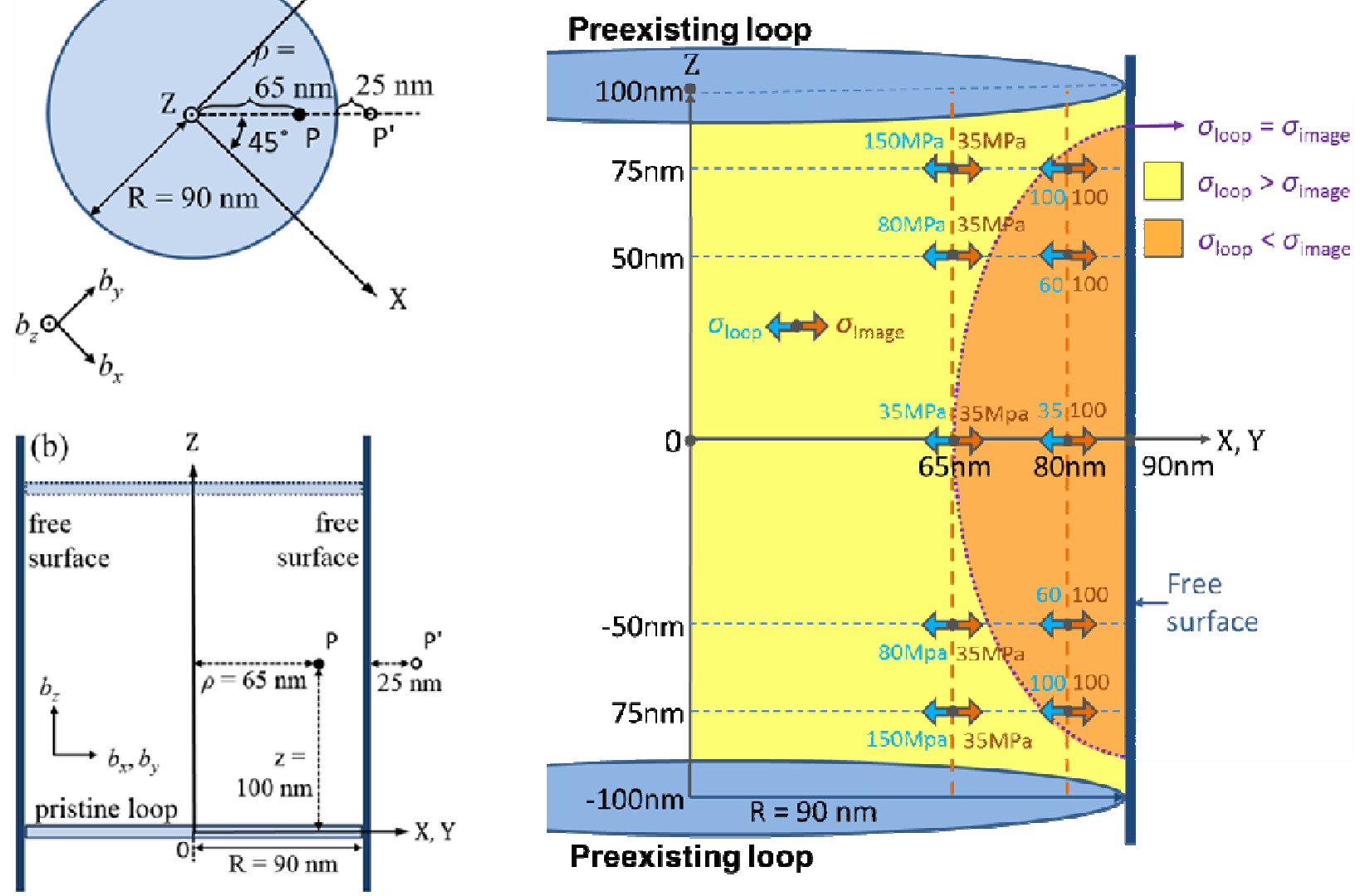

Fig.9. (a) Top view and (b) cross-section view of the geometry of two pristine dislocation loops that generate stress fields. The radius of loops is $90 \mathrm{~nm}$ and two loops were separated by $200 \mathrm{~nm}$, following the geometry in Fig. 5d. (c) Bisectional view of a nanowire with $90 \mathrm{~nm}$ in radius showing stress values at numerous points between two preexisting large loops. The stress field from the preexisting large loops is compared to that of image stress for a dislocation loop with diameter of $6 \mathrm{~nm}$. 